\title{
THE DRAZIN INVERSES OF PRODUCTS AND DIFFERENCES OF PROJECTIONS IN A $C^{*}$-ALGEBRA
}

\author{
YUAN LI
}

(Received 26 April 2007; accepted 17 October 2007)

Communicated by J. J. Koliha

Abstract

For two given projections $p$ and $q$ in a $C^{*}$-algebra, we investigate how to express the Drazin inverses of the product $p q$ and the difference $p-q$, and give applications. As a special case, we obtain the results of [C. Y. Deng, 'The Drazin inverses of products and differences of orthogonal projections', J. Math. Anal. Appl. 335 (2007) 64-71], with considerably simpler proofs.

2000 Mathematics subject classification: primary 15A09; secondary 46L99, 65F20.

Keywords and phrases: Drazin inverse, projection.

\section{Introduction}

Let $\mathcal{H}$ be a Hilbert space and $\mathcal{B}(\mathcal{H})$ be the set of all bounded linear operators on $\mathcal{H}$. Let $\mathcal{A}$ be a unital $C^{*}$-algebra with unit $e$. An element $a \in \mathcal{A}$ is said to be normal (selfadjoint) if $a a^{*}=a^{*} a\left(a=a^{*}\right)$ (see [17]). An element $p \in \mathcal{A}$ is said to be a projection if $p^{2}=p=p^{*}$. We use the notation

$$
\mathcal{P}(\mathcal{H})=\left\{P \in \mathcal{B}(\mathcal{H}): P^{2}=P=P^{*}\right\}, \quad \mathcal{P}(\mathcal{A})=\left\{p \in \mathcal{A}: p^{2}=p=p^{*}\right\} .
$$

If $a \in \mathcal{A}$, then $\sigma(a)$ and acc $\sigma(a)$ denote the spectrum and the set of all accumulation points of $\sigma(a)$, respectively; $a$ is quasipolar if $0 \notin \operatorname{acc} \sigma(a)$, and polar if it is quasipolar and 0 is at most a pole of the resolvent $R(\lambda ; a)=(\lambda e-a)^{-1}$ of $a$. In particular, $a$ is simply polar if 0 is at most a simple pole of the resolvent $R(\lambda ; a)$.

In 1958, Drazin (see [8]) introduced a different kind of a generalized inverse in associative rings and semigroups, one which does not have the reflexivity property, but commutes with the element. Let $a, b$ be elements of a semigroup. An element $b$ is the Drazin inverse of $a$, written $b=a^{D}$, if

$$
a b=b a, \quad b=b^{2} a, \quad a^{k}=b a^{k+1},
$$

This work is supported by NSF of China (No. 10726043).

(c) 2009 Australian Mathematical Society 1446-7887/2009 \$16.00 
for some nonnegative integer $k$. It is well known that if $a$ is Drazin invertible, then the Drazin inverse is unique.

In 1996, Koliha (see [12]) introduced the generalized Drazin inverse in a ring. An element $a$ of a unital ring $R$ (with unit $e^{\prime}$ ) is quasinilpotent if $e^{\prime}-x a \in \operatorname{Inv}(R)$ for every $x$ commuting with $a$, where $\operatorname{Inv}(R)$ is the set of all invertible elements of $R$. Let $a \in R$. Then an element $b \in R$ is the generalized Drazin inverse of $a$ if

$$
a b=b a, \quad b=b^{2} a, \quad a-b a^{2} \text { is quasinilpotent. }
$$

It is shown that $a$ is quasipolar if and only if $a$ is generalized Drazin invertible (see [12]). An element $a \in \mathcal{A}$ is Moore-Penrose invertible if there exists $x \in \mathcal{A}$ such that

$$
a x a=a, \quad x a x=x, \quad(a x)^{*}=a x \quad(x a)^{*}=x a .
$$

There is at most one element $x$ satisfying Equation (3); if $a \in \mathcal{A}$ is Moore-Penrose invertible, then the unique solution of Equation (3) is called the Moore-Penrose inverse of $a$ and is denoted by $a^{+}$. In recent years, a number of researchers have given much attention to the Drazin inverse and Moore-Penrose inverse in $C^{*}$-algebras (see [9, 10, 12-15]). In particular, Koliha (see [13]) found that $a \in \mathcal{A}$ is MoorePenrose invertible if and only if $a^{*} a$ (or $a a^{*}$ ) is Moore-Penrose invertible if and only if $a^{*} a$ (or $a a^{*}$ ) is quasipolar if and only if $a^{*} a$ (or $a a^{*}$ ) is simply polar. Then $a^{*} a$ (or $a a^{*}$ ) is generalized Drazin invertible if and only if $a^{*} a$ (or $a a^{*}$ ) is Drazin invertible.

In this note, the Drazin inverses of products and differences of projections in a $C^{*}$-algebra are considered. The problem of the Drazin invertibility of the sum was first considered by Drazin in his celebrated paper [8]. Some additive perturbation results for Drazin inverses are given in [1, 4, 7, 11]. In particular, the Drazin and Moore-Penrose inverses of products and differences of orthogonal projections on a Hilbert space are obtained in $[2,6]$.

The aim of this note is to present some formulae for the Drazin inverses of products and differences of projections in a $C^{*}$-algebra. As a special case, we recover theorems and corollaries of [6] which consider similar questions on a Hilbert space. The techniques used by Deng in [6] are based on complicated space decompositions and on operator matrix representations which require pages of calculations. In contrast, the methods of the present paper, based on algebraic and spectral techniques in $C^{*}$-algebras, lead to a significant simplification of arguments.

\section{Main results}

To prove the main results, we shall begin with some lemmas.

If $A$ and $B$ are $n \times n$ complex matrices, Cline's formula (see [3]) is

$$
(A B)^{D}=A\left[(B A)^{D}\right]^{2} B .
$$

For $a, b$ in an associative semigroup, if $b a$ is Drazin invertible, then it is easy to verify that the element $c=a\left((b a)^{D}\right)^{2} b$ (see [5]) satisfies the definition of the Drazin inverse 
of $a b$, so $a b$ is Drazin invertible. Similarly, if $a b$ is Drazin invertible, then $b a$ is Drazin invertible. Therefore, we have the following lemma.

Lemma 1. Let $a, b \in \mathcal{A}$. Then $a b$ is Drazin invertible if and only if ba is Drazin invertible.

Lemma 2 (see [17, Remark 1.2.1]). Let $a, b \in \mathcal{A}$. Then $\sigma(a b) \backslash\{0\}=\sigma(b a) \backslash\{0\}$.

REMARK. From Lemma 2, it is clear that $a b$ is generalized Drazin invertible if and only if $b a$ is generalized Drazin invertible.

Lemma 3. Let $p, q \in \mathcal{P}(\mathcal{A})$. Then $e-p-q$ is Drazin invertible if and only if $p q$ is Drazin invertible.

PROOF. For $p, q \in \mathcal{P}(\mathcal{H})$,

$$
(\lambda-1+p)(\lambda-p-q)(\lambda-1+q)=\lambda\left((\lambda-1)^{2}-p q\right)
$$

[16, Equation 2.5]. It is easy to verify that the equation holds for $p, q \in \mathcal{P}(\mathcal{A})$. Then for $\lambda \in \mathbb{C} \backslash\{0,1\}$, it is clear that $\lambda \in \sigma(p+q)$ if and only if $(\lambda-1)^{2} \in \sigma(p q)$.

By Lemma $1, p q$ is Drazin invertible if and only if $p q p$ is Drazin invertible. It follows from [13, Theorem 2.4] that $p q p$ is Drazin invertible if and only if $0 \notin \operatorname{acc} \sigma(p q p)$. From Lemma 2 and Equation (4), we know that $0 \notin$ acc $\sigma(p q p)$ if and only if $0 \notin \operatorname{acc} \sigma(p q)$ if and only if $1 \notin \operatorname{acc} \sigma(e-p-q)$ if and only if $e-p-q$ is Drazin invertible.

In the following, some equivalent conditions for $p q$ to be Drazin invertible are given. As a corollary, we obtain [6, Theorem 2.1].

Proposition 4. Let $p, q \in \mathcal{P}(\mathcal{A})$. Then the following statements are equivalent:

(a) $p q$ is Drazin invertible;

(b) $q p$ is Drazin invertible;

(c) $(e-p)(e-q)$ is Drazin invertible;

(d) $(e-q)(e-p)$ is Drazin invertible;

(e) $p q$ is quasipolar (or generalized Drazin invertible);

(f) pqp is Drazin invertible.

PROOF. (b) if and only if (c). It follows from Lemma 3 that $p q$ is Drazin invertible if and only if $e-p-q$ is Drazin invertible if and only if $e-(e-p)-(e-q)$ is Drazin invertible if and only if $(e-p)(e-q)$ is Drazin invertible. The proof of the rest follows from Lemmas 1 and 2.

For $P, Q \in \mathcal{P}(\mathcal{H})$, [6, Theorem 2.4] has shown the equivalences of the following Proposition 5 , by space decompositions and operator block techniques. The proof of [6, Theorem 2.4] is long and complicated. However, our proof of Proposition 5 is simple and algebraic. 
Proposition 5. Let $p, q \in \mathcal{P}(\mathcal{A})$. Then the following statements are equivalent:

(a) $p-q$ is Drazin invertible;

(b) $p+q$ is Drazin invertible;

(c) $p-q p$ is Drazin invertible;

(d) $q-q p$ is Drazin invertible.

Proof. For $p, q \in \mathcal{P}(\mathcal{H})$,

$$
(\lambda-1+p)(\lambda-p+q)(\lambda-1+q)=\lambda\left(\lambda^{2}-1+p q\right)
$$

[16, Equation 2.4]. It is also easy to verify that the equation holds for $p, q \in \mathcal{P}(\mathcal{A})$. From Equations (4) and (5), it is easy to see that $0 \in \operatorname{acc} \sigma(p-q)$ if and only if $0 \in$ acc $\sigma(p+q)$. Thus $p-q$ is Drazin invertible if and only if $p+q$ is Drazin invertible.

It follows from Lemma 3 that $q(e-p)$ is Drazin invertible if and only if $p-q$ is Drazin invertible if and only if $p(e-q)$ is Drazin invertible.

Let $a, b \in \mathcal{A}$ be Drazin invertible. It has been shown that if $a b=b a=0$, then $(a+b)^{D}=a^{D}+b^{D}$ (see [8, 12]). This leads to the following lemma.

LeMma 6. Let $p \in \mathcal{P}(\mathcal{A})$ and $a \in \mathcal{A}$ be self-adjoint and Drazin invertible. If pa $(e-$ $p)=0$ then $p a^{D}(e-p)=0$.

Proof. Since $p a(e-p)=0$, then $(e-p) a p=0$, so $a=p a p+(e-p) a(e-p)$, hence $\sigma(p a p) \backslash\{0\} \subseteq \sigma(a) \backslash\{0\}$. It follows from $0 \notin$ acc $\sigma(a)$ that $0 \notin$ acc $\sigma(p a p)$, so pap is Drazin invertible. By a similar argument, $(e-p) a(e-p)$ is Drazin invertible. Thus $a^{D}=(p a p)^{D}+[(e-p) a(e-p)]^{D}$. Since $(e-p) p a p=0$ and $(\text { pap })^{D}=($ pap $)\left[(\text { pap })^{D}\right]^{2}$, then $(e-p)(\text { pap })^{D}=0$, so $p(\text { pap })^{D}=(\text { pap })^{D}$. Similarly, $(\text { pap })^{D} p=(\text { pap })^{D}$ and

$$
[(e-p) a(e-p)]^{D}=(e-p)[(e-p) a(e-p)]^{D}=[(e-p) a(e-p)]^{D}(e-p) .
$$

Thus $a^{D}=(p a p)^{D} p+(e-p)[(e-p) a(e-p)]^{D}$, so $p a^{D}(e-p)=0$.

In the following, we present some formulae for $(p q)^{D}$, where $p, q \in \mathcal{P}(\mathcal{A})$. The results of [6, Theorem 2.2(1) and (2)] follow as special cases of (a) and (e). The result of (c) is an improvement of [6, Theorem 2.2(3)] which obtains $(P Q P)^{D} P Q=$ $(P Q)^{D} P Q$ for $P, Q \in \mathcal{P}(\mathcal{H})$.

Proposition 7. Let $p, q \in \mathcal{P}(\mathcal{A})$. If $p q$ is Drazin invertible, then the following statements hold:

(a) $(p q)^{D}=q p$ if and only if $p q=q p$;

(b) $p(p q)^{D}=(p q)^{D}$;

(c) $(p q p)^{D}=(p q)^{D} p$; 
(d) $\quad p[(e-q)(e-p)]^{D}=-(p q)^{D}(e-p)$;

(e) $(p q)^{D}=(p q p)^{D}-p[(e-q)(e-p)]^{D}$.

\section{PROOF.}

(a) Sufficiency is clear.

Necessity. If $(p q)^{D}=q p$, then $q p(p q) q p=q p$, so $(q p)^{2}=q p$. Hence $p q=$ $(p q)^{2}$, so $(p q)^{D}=p q$, then $p q=q p$, by the uniqueness of the Drazin inverse.

(b) Since $(e-p) p q=0$ and $(p q)^{D}=(p q)(p q)^{D}(p q)^{D}$, then $(e-p)(p q)^{D}$ $=0$. Hence $p(p q)^{D}=(p q)^{D}$.

(c) Using Cline's formula $(a b)^{D}=a\left[(b a)^{D}\right]^{2} b$, and letting $a=p q$ and $b=p$, then $(p q p)^{D}=p q\left[(p q)^{D}\right]^{2} p=(p q)^{D} p$.

(d) By Proposition 4, $(e-q)(e-p)$ is Drazin invertible. It follows from (b) that $p[(e-q)(e-p)]^{D}=p[(e-q)(e-p)]^{D}(e-p)$ and $-(p q)^{D}(e-p)=$ $-p(p q)^{D}(e-p)$, then

$$
\begin{aligned}
p[(e-q)(e-p)]^{D}+(p q)^{D}(e-p) & =p\left\{[(e-q)(e-p)]^{D}+(p q)^{D}\right\}(e-p) \\
& =p[(e-q)(e-p)+p q]^{D}(e-p) \\
& =p\left[e-(p-q)^{2}\right]^{D}(e-p) .
\end{aligned}
$$

It is easy to see that $p\left[e-(p-q)^{2}\right](e-p)=0$. Using Lemma 6 , we have $p\left[e-(p-q)^{2}\right]^{D}(e-p)=0$.

(e) From (c) and (d), (e) is clear.

For $P, Q \in \mathcal{P}(\mathcal{H})$, the sufficiency of the following statements is given in [6, Remark]. By simpler algebraic and spectral techniques, we obtain the equivalence of the following statements, for $p, q \in \mathcal{P}(\mathcal{A})$.

TheOREM 8. Let $p, q \in \mathcal{P}(\mathcal{A})$. If $p-q$ is Drazin invertible, then the following statements hold:

(a) $(p-q)^{D}=p-q$ if and only if $p q=q p$;

(b) $(p+q)^{D}=p+q$ if and only if $p q=q p=0$;

(c) $(p+q)^{D}=p+q-\frac{3}{2} p q$ if and only if $p q=q p$;

(d) $(p+q)^{D}=-\frac{1}{2} p+q$ if and only if $p q=p$;

(e) $(p+q)^{D}=p-\frac{1}{2} q$ if and only if $p q=q$.

PROOF. (a) If $p q=q p$, then obviously $(p-q)^{3}=p-q$, so it is easy to verify that $(p-q)^{D}=p-q$.

Conversely, if $(p-q)^{D}=p-q$, then $(p-q)^{3}=p-q$, so $p q p=q p q$. Hence $(p q)(p q)^{*}=p q p=q p q=(p q)^{*}(p q)$, so $p q$ is normal. Since $\sigma(p q) \subseteq[0,1]$, then by the Gelfand representation theorem [17, Theorem 2.1.10], $p q$ is self-adjoint. Hence $p q=q p$.

(b) Sufficiency is clear.

Necessity. If $(p+q)^{D}=(p+q)$, then $(p+q)^{3}=(p+q)$. By a direct calculation, $p q p+q p q=-2(p q+q p)$, so $p q+q p \leq 0$. Then $p(p q+q p) p \leq 0$, hence $p q p \leq 0$, so $p q p=0$. Then $p q=0$, so $p q=q p=0$. 
(c) If $p q=q p$, then it is easy to verify that $(p+q)^{D}=p+q-(3 / 2) p q$.

If $(p+q)^{D}=p+q-(3 / 2) p q$, then

$$
(p+q)\left(p+q-\frac{3}{2} p q\right)=\left(p+q-\frac{3}{2} p q\right)(p+q)
$$

and, by a direct calculation, $p q p=q p q$. It follows from the proof of (a) that $p q=q p$.

(d) Sufficiency is clear, by (c).

If $(p+q)^{D}=-\frac{1}{2} p+q$, then

$$
(p+q)\left(-\frac{1}{2} p+q\right)=\left(-\frac{1}{2} p+q\right)(p+q)
$$

and, by a direct calculation, $p q=q p$. Since $p+q$ is self-adjoint, then $(p+q)^{D}=$ $-\frac{1}{2} p+q$ implies that

$$
(p+q)\left(-\frac{1}{2} p+q\right)(p+q)=p+q .
$$

It follows that $2 p q p+q p+p q-q p q=3 p$, then $p q p=p$, since $p q=q p$. Hence $p q=p q p=p$. The proof of (e) is similar to that of $(\mathrm{d})$.

Let $p, q \in \mathcal{A}$ be projections. We denote by $S_{(m, p)}$ and $S_{(n, q)}$ an $m$-factor product of $p$ and $q$ with first factor $p$ and an $n$-factor product of $p$ and $q$ with first factor $q$; in both cases $p$ and $q$ occur alternately. The result of [6, Corollary 2.3] follows as a special case of the following result.

COROllary 9. Let $p, q \in \mathcal{P}(\mathcal{A})$. Then the following statements are equivalent:

(a) $S_{(m, p)}^{D}=S_{(n, q)}^{D}$ for some $m, n \geq 1$;

(b) $p q=q p$;

(c) $S_{(m, p)}^{D}=S_{(n, q)}^{D}$ for every $m, n \geq 1$.

Proof. (a) implies (b). Without loss of generality, we assume that $m \leqslant n$. Obviously, $S_{(m, p)}^{D}=S_{(n, q)}^{D}$ implies that $\left[(p q)^{D}\right]^{m}=\left[(q p)^{D}\right]^{n}$. Since $p(p q)^{D}=$ $(p q)^{D}=(p q)^{D} q$, we have

$$
\left[(p q)^{D}\right]^{m}=(p q)^{D} p(p q)^{D} \ldots p(p q)^{D}=\left[(q p)^{D}\right]^{n},
$$

so

$$
\left[(p q)^{D}\right]^{m} p=(p q)^{D} p(p q)^{D} \ldots p(p q)^{D} p=\left[(q p)^{D}\right]^{n} p=\left[(q p)^{D}\right]^{n} .
$$

It follows from $(p q)^{D} p=(p q p)^{D}$ that $\left[(p q)^{D}\right]^{m}=\left[(p q p)^{D}\right]^{m}$, so $\left[(p q)^{D}\right]^{m}$ is selfadjoint, then $\left[(p q)^{D}\right]^{m}=\left[(q p)^{D}\right]^{m}$. Similarly,

$$
\left[(p q p)^{D}\right]^{m}=\left[(p q)^{D}\right]^{m}=\left[(q p)^{D}\right]^{m}=\left[(q p q)^{D}\right]^{m},
$$

then $(p q p)^{D}=(q p q)^{D}$. Therefore,

$$
p q p=\left[(p q p)^{D}\right]^{D}=\left[(q p q)^{D}\right]^{D}=q p q .
$$

As in the proof of Theorem 8(a), $p q=q p$.

(b) implies (c) and (c) implies (a) are clear. 
In the following, we present two formulae for $(p-q)^{D}$ and $(p+q)^{D}$ where $p, q \in \mathcal{P}(\mathcal{A})$. As a corollary of formula (a), we obtain [6, Theorem 2.5(3)]. Formula (b) is an improvement of [6, Theorem 2.5(4)] which leads to

$$
P\left[(P+Q)^{D}-(P-Q)^{D}\right](P-Q)^{2}=0,
$$

for $P, Q \in \mathcal{P}(\mathcal{H})$.

THEOREM 10. Let $p, q \in \mathcal{P}(\mathcal{A})$. If $p-q$ is Drazin invertible, then the following statements hold:

(a) $(p-q)^{D}=(p-q)^{2}\left[(p-q p)^{D}-(q-q p)^{D}\right]$;

(b) $\quad p\left[(p+q)^{D}-(p-q)^{D}\right](p-q)=0$.

PROOF. (a) By Proposition 5, $p-q p$ and $q-q p$ are Drazin invertible. It is clear that

$$
\begin{aligned}
& (p-q)^{2}\left[(p-q p)^{D}-(q-q p)^{D}\right] \\
& \quad=(p-q)(p-p q+p q-q)\left[(p-q p)^{D}-(q-q p)^{D}\right] \\
& \quad=(p-q)[p(e-q)-(e-p) q]\left\{[(e-q) p]^{D}-[q(e-p)]^{D}\right\} .
\end{aligned}
$$

It follows from Proposition 7(b) that $p(e-q)[q(e-p)]^{D}=0$ and $(e-p) q[(e-$ q) $p]^{D}=0$, hence

$$
\begin{aligned}
(p-q)^{2}\left[(p-q p)^{D}-(q-q p)^{D}\right]= & (p-q)\left\{p(e-q)[(e-q) p]^{D}\right. \\
& \left.+(e-p) q[q(e-p)]^{D}\right\} .
\end{aligned}
$$

Using Proposition 7(c) again,

$$
\begin{aligned}
(p-q)^{2} & {\left[(p-q p)^{D}-(q-q p)^{D}\right] } \\
& =(p-q)\left\{[p(e-q) p]^{D}+[(e-p) q(e-p)]^{D}\right\} \\
& =(p-q)\left\{[p(e-q) p+(e-p) q(e-p)]^{D}\right\} \\
& =(p-q)\left[(p-q)^{2}\right]^{D} \\
& =(p-q)(p-q)^{D}(p-q)^{D} \\
& =(p-q)^{D} .
\end{aligned}
$$

(b) To show this, we need the following lemmas.

LEMMA 11. Let $p, q, w \in \mathcal{P}(\mathcal{A})$. If $(p+q) w=0$, then $p q w=0$.

PROOF. $(p+q) w=0$ implies that $p w p+q w p=0$ and $(e-p) q w=0$. Thus $q w=$ $p q w$ and $q w p$ is self-adjoint. Since

$$
\sigma(q w p) \backslash\{0\}=\sigma(p q w) \backslash\{0\}=\sigma(q w) \backslash\{0\}=\sigma(q w q) \backslash\{0\} \subseteq[0,1],
$$

then $\sigma(q w p) \subseteq[0,1]$, so $q w p \geq 0$. Hence $p w p=0$, then $p w=0$, so $q w=0$. It follows that $p q w=0$. 
LEMMA 12 (see [13]). Let $a \in \mathcal{A}$ be self-adjoint. Then:

(a) a is Moore-Penrose invertible if and only if a is Drazin invertible;

(b) if a is Drazin invertible, then $a^{+}=a^{D}$, and $a^{2} a^{D}=a$.

The following identity was first obtained by Wedin (see [18]) for matrices and by Harte and Mbekhta (see [10, Theorem 5]) for $C^{*}$-algebras.

Lemma 13. Let $a, b \in \mathcal{A}$ be Moore-Penrose invertible. Then

$$
\begin{aligned}
b^{+}-a^{+}= & -b^{+}(b-a) a^{+}+\left(e-b^{+} b\right)\left(b^{*}-a^{*}\right)\left(a^{+}\right)^{*} a^{+} \\
& +b^{+}\left(b^{+}\right)^{*}\left(b^{*}-a^{*}\right)\left(e-a a^{+}\right) .
\end{aligned}
$$

Proof of (b) of Theorem 10. By Proposition 5, $p+q$ is Drazin invertible. For convenience, let $b=p+q$ and $a=p-q$. Then by Lemmas 12 and 13,

$$
\begin{aligned}
b^{D}-a^{D} & =b^{+}-a^{+} \\
& =-b^{D}(2 q) a^{D}+\left(e-b^{D} b\right)(2 q)\left(a^{D}\right)^{2}+\left(b^{D}\right)^{2}(2 q)\left(e-a a^{D}\right),
\end{aligned}
$$

so

$$
\left(b^{D}-a^{D}\right) a=-b^{D}(2 q) a^{D} a+\left(e-b^{D} b\right)(2 q) a^{D},
$$

and hence

$$
\begin{aligned}
& p\left[(p+q)^{D}-(p-q)^{D}\right](p-q) \\
& \quad=p\left[2 q(p-q)^{D}-2(p+q)^{D} q(p-q)^{D}(p-q)\right. \\
& \left.\quad-2(p+q)^{D}(p+q) q(p-q)^{D}\right] .
\end{aligned}
$$

Since

$$
(p+q)(q p+p q)=(q p+p q)(p+q),
$$

it follows from [12, Theorem 4.4] that

$$
(p+q)^{D}(q p+p q)=(q p+p q)(p+q)^{D} .
$$

Therefore,

$$
\begin{aligned}
p[(p & \left.+q)^{D}-(p-q)^{D}\right](p-q) \\
& =2 p\left\{q(p-q)^{D}-(p+q)^{D}[q(p-q)+(p+q) q](p-q)^{D}\right\} \\
& =2 p\left[q(p-q)^{D}-(p+q)^{D}(q p+p q)(p-q)^{D}\right] \\
& =2\left[p q(p-q)^{D}-p(q p+p q)(p+q)^{D}(p-q)^{D}\right] \\
& =2\left[p q(p-q)^{D}-p(q p+p q)(p+q)^{D}(p-q)^{D}\right] \\
& =2\left[p q(p-q)^{D}-p q(p+q)(p+q)^{D}(p-q)^{D}\right] \\
& =2\left\{p q\left[e-(p+q)(p+q)^{D}\right](p-q)^{D}\right\} .
\end{aligned}
$$


Note that

$$
e-(p+q)(p+q)^{D} \in \mathcal{P}(\mathcal{A})
$$

and

$$
(p+q)\left[e-(p+q)(p+q)^{D}\right]=0
$$

then, by Lemma 11 ,

$$
p q\left[e-(p+q)(p+q)^{D}\right]=0
$$

so

$$
p\left[(p+q)^{D}-(p-q)^{D}\right](p-q)=0 .
$$

\section{Acknowledgement}

The author would like to express his hearty thanks to the referee for his/her valuable comments and suggestions on the presentation of this paper.

\section{References}

[1] G. Castro and J. J. Koliha, 'New additive results for the g-Drazin inverse', Proc. Roy. Soc. Edinburgh Sect. A 134 (2004), 1085-1097.

[2] S. Cheng and Y. Tian, 'Moore-Penrose inverses of products and differences of orthogonal projectors', Acta Sci. Math. (Szeged) 69 (2003), 533-542.

[3] R. E. Cline, 'An application of representation of a matrix', MRC Technical Report 592 (1965).

[4] D. S. Cvetković-Ilić, D. S. Djordjević and Y. Wei, 'Additive results for the generalized Drazin inverse in a Banach algebra', Linear Algebra Appl. 418 (2006), 53-61.

[5] A. Dajic and J. J. Koliha, 'The weighted $g$-Drazin inverse for operators', J. Aust. Math. Soc. 82 (2007), 163-181.

[6] C. Y. Deng, 'The Drazin inverses of products and differences of orthogonal projections', J. Math. Anal. Appl. 335 (2007), 64-71.

[7] D. S. Djordjević and Y. Wei, 'Additive results for the generalized Drazin inverse', J. Aust. Math. Soc. 73 (2002), 115-125.

[8] M. P. Drazin, 'Pseudoinverse in associative rings and semigroups', Amer. Math. Monthly 65 (1958), 505-514.

[9] R. E. Harte and M. Mbekhta, 'On generalized inverses in $C^{*}$-algebras', Studia Math. 103 (1992), 71-77.

[10] - 'Generalized inverses in $C^{*}$-algebras II', Studia Math. 106 (1993), 129-138.

[11] R. E. Hartwig, G. R. Wang and Y. Wei, 'Some additive results on Drazin inverse', Linear Algebra Appl. 322 (2001), 207-217.

[12] J. J. Koliha, 'A generalized Drazin inverse', Glasgow Math. J. 38 (1996), 367-381.

[13] _ 'The Drazin and Moore-Penrose inverse in $C^{*}$-algebras', Math. Proc. R. Ir. Acad. 99A (1999), 17-27.

[14] - 'Elements of $C^{*}$-algebras commuting with their Moore-Penrose inverse', Studia Math. 139 (2000), 81-90.

[15] — 'Continuity and differentiability of the Moore-Penrose inverse in $C^{*}$-algebras', Math. Scand. 88 (2001), 154-160. 
[16] J. J. Koliha and V. Rakočević, 'Fredholm properties of the difference of orthogonal projection in a Hilbert space', Integral Equations Operator Theory 52 (2005), 125-134.

[17] G. J. Murphy, $C^{*}$-Algebras and Operator Theory (Academic Press, New York, 1990).

[18] P. A. Wedin, 'Perturbation theory for pseudo-inverses', BIT 13 (1973), 217-232.

YUAN LI, College of Mathematics and Information Science, Shaanxi Normal University, Xi' an 710062, People's Republic of China e-mail: liyuan0401@yahoo.com.cn 